\title{
Differential neural activity patterns mediate learning across contexts in a social cichlid fish
}

${ }^{1}$ Institute for Neuroscience, ${ }^{2}$ Department of Integrative Biology, ${ }^{3}$ Institute for Cell and Molecular

Animal Behavior, Konstanz, Germany

Mariana Rodriguez-Santiago ORCID: 0000-0002-4919-6417

Alex Jordan ORCID: 0000-0001-6131-9734

Hans A. Hofmann ORCID: 0000-0002-3335-330X

Corresponding author:

18 Dr. Mariana Rodriguez-Santiago

Department of Biology

W. Lake Street

Colorado State University

22 Fort Collins, CO 80523

23 Phone: 303-324-5208

24 Email: mari.rodriguez221@gmail.com

Corresponding author:

27 Dr. Hans A. Hofmann

28 Department of Integrative Biology

292415 Speedway - C0930

30 Austin, TX 78712, USA

31 Phone: 512-475-6754

32 Email: hans@utexas.edu

Word Count: abstract: 188; main text: 4574

Figures: 3

37

Key words: animal behavior, cichlid fish, neural activity, social learning 


\section{Abstract}

40 Learning and decision-making are greatly influenced by the social context surrounding

41 individuals. When navigating a complex social world, individuals must quickly ascertain where

42 to gain important resources and which group members are useful sources of such information.

43 Such dynamic behavioral processes require neural mechanisms that are flexible across contexts.

44 Here we examined how the social context influences the learning response during a visual cue

45 discrimination task and the neural activity patterns that underlie acquisition of this novel

46 information. Using the cichlid fish, Astatotilapia burtoni, we show that learning of the task is

47 faster in social groups than in a non-social context. We quantified the expression of Fos, an

48 immediate-early gene, across candidate brain regions known to play a role in social behavior and

49 learning, such as the putative teleost homologues of the mammalian hippocampus, basolateral

50 amygdala, and medial amygdala/BNST complex. We found that neural activity patterns differ

51 between social and non-social contexts. Our results suggest that while the same brain regions

52 may be involved in the learning of a discrimination task independent of social context, activity in

53 each region encodes specific aspects of the task based on context. 


\section{Introduction}

For group-living species, social interactions provide a key source of information that can greatly impact the fitness and well-being of individual group members. It is commonly assumed that learning from others, or social learning, is inherently adaptive as it allows individuals to avoid costs associated with learning by themselves, or non-socially [1]. The benefits of social learning allow individuals to gain information from conspecifics, such as to which foods to eat, which routes to take to feeding locations, and how to escape from predators [2]. These wideranging behaviors have been studied across species, such as in instances of socially transmitted

62 food preferences [3, 4], social learning of certain skills $[5,6,7]$, mate preference learning

63 [reviewed in 8], predator avoidance [9], and fear transmission [10, reviewed in 11]. The

64 behavioral mechanisms that underlie these behaviors are diverse, ranging from stimulus enhancement (when another individual draws the observer's attention to a particular stimulus or object) to observational learning $[12,13,14]$, allowing animals to acquire new information important for their survival and which can incidentally be transmitted to conspecifics $[15,16,17$, 18]. While a lot is known about the neural basis of learning in non-social contexts [reviewed in 19], few studies have examined whether and how these mechanisms might operate in the context of social learning.

Studies in rodents and songbirds have expanded our understanding of the neurobiological mechanisms that mediate social learning, such as the brain regions that are important for

73 acquisition and maintenance of socially-transmitted food preferences in rats [reviewed in 20].

74 Subregions of the hippocampus (specifically, the subiculum and dentate gyrus) have been shown 
fear has been found to be also modulated in part by the lateral nucleus of the amygdala in both

77 rhesus monkeys and humans $[11,24]$.

At the molecular level, social learning requires neural activity-dependent changes in gene expression, much like long-lasting alterations in the strength of synaptic connectivity important for associative learning [25, 26]. Activation of immediate early genes (IEGs) is a critical mediator in this process $[27,28]$. Previous studies in rodents have shown that IEGs such as $c f o s$ are expressed following acquisition and consolidation of associative learning [29 - 32]. In addition, rats trained on a test of social transmission of food preference show greater $c f o s$ expression in subregions of the hippocampus in a time-dependent manner [29, 30]. The medial amygdala plays a key role in mouse social cognition, as oxytocin receptors in this region are essential for recognizing familiar conspecifics [33]. In songbirds, differential Fos expression has

87 been shown to underlie different aspects of song learning and production [34, 35]. There is also

88 evidence in songbirds that differential neural activity underlies different phases of sexual

89 imprinting, a type of social learning by which a juvenile learns specific characteristics of a parent or other familiar individual [36]. Taken together, these findings suggest that across species

91 associative learning in social contexts is driven by differential neural activity patterns across 92 multiple brain regions.

94 learning in a model system that readily forms naturalistic social groups in the laboratory. The 95 African cichlid fish, Astatotilapia burtoni, is a model system in social neuroscience because of 96 its remarkable phenotypic plasticity and sophisticated social cognition $[37,38]$. Dominant males

97 of this species are territorial and aggressive, while subordinates typically do not hold territories 98 and are overall less aggressive [38, 39]. In a recent study, we found that although dominant 
males of this species had strong influence over the movement of their social groups under normal conditions, they were less influential in a more complex learning task [40]. This effect was primarily driven by the socially aversive behavior of dominant males, which, although central in interaction networks, occupied peripheral positions in spatial networks. IEG expression in response to different types of social information has also been shown in this species [41 - 44], suggesting that differences in learning in social or non-social contexts may induce differential patterns of neural activity.

We examined IEG expression in different brain regions of $A$. burtoni males and females during learning in social groups or without a conspecific informant. We first compared the learning response rates in a social and non-social context as measured by the latency to acquire a cue association. We hypothesized that social facilitation mechanisms would allow groups to learn the task faster than individuals in the non-social context. To understand how the brain acquires a cued association across social contexts, we then quantified expression of Fos, an IEG, across the putative teleost homologues of the mammalian hippocampus, basolateral amygdala, and medial amygdala/bed nucleus of the stria terminalis (BNST) complex, which are key nodes

114 of the Social Decision-Making Network (SDMN) [45, 46]. We predicted that neural activity 115 during learning in a social context would be highest in brain regions important for mediating 116 social behavior in this species, such as the supracommissural part of the ventral pallium (Vs, the 117 putative homologue of the mammalian medial amygdala/BNST complex) and the medial part of 118 the dorsal telencephalon (Dm, the putative homologue of the basolateral amygdala); as well as 119 those important for associative learning, such as specific sub-regions of the lateral part of the 120 dorsal telencephalon (D1, the putative homologue of the hippocampus). In addition, we expected 121 neural activity in Dl to increase in both contexts once learning occurs. Finally, we predicted that 
122 neural activity in regions important for social behavior would be relatively low in the non-social

123 context. Our results reflect differences in how new information is acquired in different social

124 contexts.

125

126 2. Methods

127 Animals

Astatotilapia burtoni descended from a wild caught stock population were kept in stable

129 naturalistic communities of eight males and eight females, as described previously [46] until

130 being transferred to experimental aquaria. Brooding females were stripped of fry immediately

131 prior to being placed in experimental aquaria. All work was done in compliance with the

132 Institutional Animal care and Use Committee at The University of Texas at Austin. All relevant

133 code and analyses are available online at https://github.com/neuromari/neuro_social_learning.

Visual cue discrimination task

Our protocol broadly followed that of Rodriguez-Santiago, Nührenberg et al. (2020). A

137 detailed description of the task setup, task training in a social and non-social context, as well as

138 the response criterion we used to consider a task to have been completed successfully is provided

139 in the Supplemental Materials. Because A. burtoni communities form social dominance

140 hierarchies, we accounted for social hierarchy dynamics and group behavior in the social

141 context, as described in Supplemental Materials. 
To examine neural activity patterns across learning trials, three individual samples were

145

146

147

148

149

150

151

152

153

154

155

156

157

158

159

160

161

162

163

164

165

166

collected from each community. In groups with a dominant male informant, the second largest

male, subordinate male, and a female were collected. In groups with a subordinate male

informant, the dominant male, and third largest male, and a female were collected. For all non-

socially trained individuals, males and females were euthanized after trials 6,14 , or 22 . A

detailed description of the immunohistochemical procedures and the quantification of Fos-

positive cells is provided in the Supplemental Materials.

\section{Statistical analysis}

All statistical analyses were conducted using R Studio (version 1.0.143) and the ‘survival' package [47]. We analyzed the learning response using a survival analysis. We used the nonparametric log-rank test because the proportional hazard assumption was not met, given that it does not support multiple response variables, such as social context, informant status, and individual sex. Thus, we used a series of log-rank tests to examine the overall effect of social context and pairwise differences between informant status in the social context and sex in the non-social context. In a separate analysis, we examined differences between informant status effects in a social context, as well as sex differences in response rate in the non-social context using repeated measures analysis of variance (ANOVA).

We used Principal Components Analysis (PCA) to identify how neural activity patterns across brain regions clustered based on social context conditions and individual-level traits. Independent ANOVA tests were used to compare PC scores between social condition (social v non-social), trial, and learning response. To account for repeated measures of the same fish across treatments, generalized linear mixed models (GLMM) were used for Fos expression 
167

168

169

170

171

172

173

174

175

176

177

178

179

180

181

182

183

184

185

186

187

188

analyses, which are a proxy for neural activity. To examine how learning context, trial, and individual-level traits influence learning and neural activity patterns, we used the $\mathrm{R}$ package glmmTMB which ranks models based on Akaike Information Criterion scores, corrected for sample size (AICc) [48], and allows for usage of the beta family, which is appropriate for modeling proportional data. We first performed an overall GLMM that included both social and non-social learning conditions, and also did a separate model on the social and non-social conditions. In the overall model, we included learning condition, trial, sex, whether the learning criterion was met, and group as dependent variables and brain region (Dl-g, Dl-v, Dm-1, Dm-3, and Vs) as the independent variables. In the social condition model, the dependent variables were trial, sex, observer status, informant status, whether the learning criterion was met, and group. In the non-social condition model, the dependent variables were trial, sex, and learning as the dependent variables. Model results and tables can be found in the Supplemental Materials.

\section{Results}

Social facilitation results in faster response rates compared to a non-social context

We first asked whether the cumulative response rate differed between the social and nonsocial contexts and found that the cumulative probability of consecutive group responses during the cue discrimination task is significantly greater than the response rate of individuals in an nonsocial context (log-rank test: $X^{2}=8.1, P=0.004$; Figure 1a). However, the number of trials it took to reach the response criterion did not differ between the social and non-social contexts (Wilcox test: $\mathrm{W}=41, p=0.426$; Figure $1 \mathrm{~b}$ ). To our surprise, the social status of the informant - 
189 dominant vs. subordinate - did not have any effect on learning rate (log-rank test: $X^{2}=0.005, P$

$190=0.94)$, contrary to our previous study [40].

Neural activity patterns depend on the social context

We used PCA to determine which aspects of the social context and individual-level traits

194 influence neural activity patterns during a learning task, and how these contextual aspects

contribute to a learning response. We first conducted a PCA that included variables in both social

conditions: the trial at which individuals were taken (trial), the context condition (social v non-

social), and whether the response criterion was met (yes or no). We found that principal social conditions across trials (Figure 2$)$. There was a main effect of both social context $\left(\mathrm{F}_{1,91}=\right.$

$207=.3$; trial: $\mathrm{F}_{2,91}=76.24, p<.001$; interaction: $\mathrm{F}_{2,91}=19.45, p<.001 ;$ Figure $2 \mathrm{~h}$ : learning

208 response: $\mathrm{F}_{1,91}=59.61, p<.001$; trial: $\mathrm{F}_{2,91}=26.16, p<.001$; trial $\mathrm{x}$ learning response: $\mathrm{F}_{2,91}=$

$209.064, p=.938$; Figure 2i: learning response: $\mathrm{F}_{1,91}=47.93, p<.001$; context: $\mathrm{F}_{1,91}=13.493, p=$ 
212 conducted separate PCAs on the social (Supplemental Figure 5) and non-social contexts. The

213 results of these analysis can be found in the Supplemental Materials and Figures.

Neural activity patterns during acquisition of learning differ across social contexts

217 learning context, we examined neural activity in key nodes of the SDMN across trials and activity across social conditions (social, non-social) and learning task trial $(6,14,22)$ using twointeraction. In the Dm-1, there was both a significant main effect of trial and context as well as examined neural activity in the Dl-v and the Dm-3 subregions and found no significant effect of statistics). Across all brain regions, there was a main effect of context and learning response.

231 There was an interaction between learning and context in the Dm-1 only. There was no

232 difference in Fos expression in the non-social context based on whether individuals learned the 233 task, while in the social context Fos expression was highest in observers that learned the task in 234 the Dl-g ( $p<0.001$; Figure $3 \mathrm{~b})$ as well as in the Vs $(p=0.001$; Figure 3f). Despite the large 

necessarily surprising given the prevalence of social learning strategies across species and the

differences between Fos expression across social contexts in the five brain regions measured over trials, when we looked closer at factors that impact this difference within the social context we found no significant differences in expression based on the social rank of observers or based on the informant status (data not shown).

\section{Discussion}

In the present study, we found large differences between a social and non-social context in behavioral and neural activity during an associative learning task. Specifically, we discovered a significant difference in learning rate between contexts, such that social groups had a higher cumulative probability of reaching the response criterion sooner than individuals in a non-social context. This striking behavioral difference is reflected in the neural activity pattern differences between contexts, with specific brain regions encoding different aspects of our learning paradigm, suggesting that the acquisition of a learning response to a cue association is mediated by different brain regions depending on the social context.

\section{Observational learning and stimulus enhancement accelerate associative learning of a visual}

\section{cue discrimination task}

By examining the cumulative learning rate probability of acquiring a cue association

$$
\text { response across two experimental contexts, we found that social groups had a significantly }
$$

higher cumulative probability of learning than individuals in a non-social context. This is not

257 notion that social learning is more adaptively beneficial as it confers fewer costs and allows 
individuals to gain new information more quickly [49]. In addition, information diffusion is typically accelerated in social groups [20].

There are at least two mechanisms by which learning might have occurred in our social paradigm, social facilitation (when the presence of an informant affects the observer's behavior) and stimulus enhancement (where the observer's behavior changes after watching an informant interact with a stimulus). To demonstrate that the group response is due solely to the presence of an informant (i.e., social facilitation), it would be necessary to test individual group members by themselves following acquisition. While we did not examine this retention by observers in the present study, it should be noted that the informants themselves were trained in naïve groups and then transplanted to new groups, where they were the only informed individual. Importantly, all

268 informants displayed a correct response to the cue within one or two trials in their new

269 communities, suggesting that the observers in our study in fact acquired the association and were 270 not just copying other group members' behavior. It seems thus likely that individuals in social 271 groups learned by means of observation or stimulus enhancement, which ultimately led them to 272 respond faster than lone individuals. However, it cannot be ignored that A. burtoni is a highly 273 social species, and although individuals in the non-social context had blind cave fish as a social 274 buffer, their slow learning rate could be due to stress factors from being apart from conspecifics. 
281 putative homologue of the mammalian hippocampus), we found a significant increase in Fos

282 expression (or 'activity') from trial 6 to trial 14 in the social context in the Dl-g sub-region,

283 which was also significantly higher in groups that learned the task. In the Dl-v sub-region, there

284 was a significant main effect of social context across trials, and a significant decrease in activity

285 between trials 14 and 22. Activity in the Dl-v was not correlated with learning. The Dl-g and Dl-

$286 \mathrm{~V}$ are subdivisions of the dorsal pallium, a region implicated in the learning of spatial and

287 temporal relationships in teleosts $[50,51]$. Previous work has also shown that the major

288 pathways within the dorsal pallium are highly recursive and have complex reciprocal

289 connections with subpallial regions [52]. Based on tract-tracing neuroanatomical data, as well as

290 lesions studies that implicate the $\mathrm{Dl}$ and other dorsal pallial regions in learning and memory

291 tasks, Elliott et al. (2016) suggested that the dorsal pallial circuitry (which includes the D1

292 subregions) can implement the same pattern separation and completion computations ascribed to

293 the mammalian hippocampal dentate gyrus and CA3 fields. Taken together, these results suggest

294 a differential role for these Dl subregions in the acquisition of this association learning task.

296 The basolateral amygdala likely encodes social group formation, not learning of the association

297 task

We found a significant difference in neural activity across social contexts in subregions

299 of Dm (medial part of the dorsal telencephalon and putative homologue of the mammalian

300 basolateral amygdala). More specifically, we found a significant decrease in activity across trials

301 in the social context in Dm-1, and a significant decrease from trial 14 to 22 . Activity in Dm-1

302 was not associated with group learning, and there was no difference across learning response in

303 the Dm-3 (not shown). These findings are consistent with previous studies in goldfish that have 
shown that Dm lesions disrupt trace and delay avoidance conditioning [51, 53], as well as fear and heart-rate classical conditioning [22], while such lesions have no effect on spatial memory and cue learning $[54,55]$. The effects of these lesions in fish are similar to lesions of the amygdala in mammals [56 - 59] and in part based on this evidence the teleost medial pallium defense, although the sign of the correlation depends on an individual's role in this cooperative behavior [44]. In the present study, we found that activity in the Dm complex was significantly higher in trial 6 compared to 14 and 22. Given that few groups had learned the task prior or by

314 learned the task. Interestingly, individuals from groups that did reach the learning criterion by trial 6 or sooner showed lower Dm activity, which further indicates that Dm is not involved in

316 learning the cue association task. Instead, this result suggests that the Dm regions, and the Dm-1

317 in particular, may play a role in some aspect of social group formation rather than being involved in the acquisition of the cue association task, providing further support for a role of this brain region in affective processing.

The extended medial amygdala encodes social context

323 mammalian medial amygdala/BNST) we found a significant main effect of social context. Also,

324 Vs activity increased in social groups in trials 14 and 22, possibly as a consequence of more

325 groups successfully learning the task at these later trials. Homology of this brain region has

326 historically been difficult to characterize due to the eversion, rather than invagination, of the 
327

328

329

330

331

332

333

neural tube during teleost development [60 - 63]. However, developmental studies have found similar genetic markers, namely $D l x 2, L h x 7, N k x 2.1 b$, between the Vs and the extended amygdala [64]. Stimulation of the Vs has been shown to increase aggression in male bluegill fish [65]. In our species, A. burtoni, this region is under social and reproductive modulation [42] and shows varying levels of sex steroid receptor expression in males when given the opportunity to ascend or descend in status. Taken together, this suggests that Vs plays a predominant role in mediating social information, which is why we see large differences in neural activity here between the social and non-social learning contexts.

\section{Disentangling the effects of group formation and learning on neural activity patterns}

While we see evidence for differential neural activity across multiple brain regions during the acquisition of an association in both social and non-social contexts, we are unable to fully separate the effects of group formation time from the effects of learning. Even though there are significant differences in neural activity in specific brain regions (Dl, Dm) based on whether groups demonstrated learning, it remains unclear how group formation impacts learning. In other words, there could be a dampening of response in early trials due to social instability simply because the groups did not have time to acclimate prior to the start of the trials. In the non-social context, we observed a general dampening of neural activity specifically in early trials that coincided with lower behavioral activity levels. Disentangling the effects of social stability formation from the increased probability of learning after repeated trials in both social and nonsocial contexts will require subsequent rigorous behavioral examination with automated tracking.

\section{What Fos expression tells us about the observed neural activity patterns}


An important aspect of examining IEG induction as a measure of neural activity is that we examined this expression 1 hour after the last learning trial the animals underwent - whether activity $[66,67]$ as most IEGs encode transcription factors or DNA-binding proteins that coordinate the cellular response to a stimulus [28]. By examining Fos protein expression within 60-90 minutes following the last stimulus exposure, we aimed to capture the brain regions that were active, and presumably important, for the animal's behavioral response. Animals did not

357 perform these behaviors in isolation, and it is possible that both in the social and non-social

358 contexts their neural activity reflects a response to the environment rather than the stimulus cue itself. For example, there could have been a salient social signal occurring in the aquarium at the same time as the cue (such as high territorial aggression by a dominant male). However, given

365 although the aggressive behaviors of other observer males could have had an effect on the neural activity patterns seen in the social context.

Communities of A. burtoni naturally form rank hierarchies with some males establishing social dominance by aggressively defending territories for mating with females, while the

371 majority of males are socially subordinate and reproductively suppressed [37, 68]. We have

372 previously shown for this species that the social status of an informant can have a strong effect 
373 on how fast a group learns the visual cue discrimination task we used in the present study.

374 Specifically, even though socially dominant males strongly influence their social groups through

375 aggressive displays and space use, they are significantly less effective in generating group

376 consensus during the association task than subordinate males [40]. In contrast, we did not find a

377 significant effect of informant status on group learning in the present study. This may not be

378 surprising given that the present study was not designed to examine the effects of social status on

379 group learning, and thus lacks the statistical power to robustly detect such an effect. It should

380 also be noted that in the Rodriguez-Santiago, Nührenberg et al. (2020) study, dominant males

381 were considerably larger than subordinate males, while in the present study the size difference

382 was much smaller. Previous work has shown that small size differences result in lower stability

383 of the social hierarchy in this species [69]. Although we did not quantify group stability here, the

384 behavioral traits that determine whether an individual is an effective informant - aggression and

385 space use - are highly context-specific and might explain the absence of a social status effect.

386 These factors may also explain why we did not find differences in neural activity patterns

387 between dominant and subordinate observers when learning the task. One interesting observation

388 of relevance here comes from social fear learning in rats, where subordinate animals display

389 increased fear responses after interacting with a dominant informant, which is also reflected in

390 distinct neural activity patterns [70].

\section{Conclusion}

We used the highly social African cichlid fish A. burtoni to demonstrate that social

395 learning is associated with increased neural activity (as measured by the expression of Fos, an 
IEG) when compared to non-social learning across key brain regions important for learning and

397 social behavior. These brain regions are important for modulating learning (hippocampus),

398 emotional learning and fear avoidance (basolateral amygdala), and social behavior (medial

399 amygdala/BNST), and are part of a greater Social Decision-Making Network that is important

400 for mediating various aspects of social behavior $[45,46]$. In addition, we found that activity in

401 these regions was not modulated by the sex or social status of individuals, nor was it impacted by

402 the status of informants in social groups. Thus, while these regions are important for different

403 aspects of social learning [45], they do not appear to be modulated by group dynamics or

404 individual-level traits in a social learning context. While future studies are needed to fully

405 understand the mechanisms that drive social learning contexts (e.g. neuroendocrine or

406 dopaminergic pathways), our results in A. burtoni highlight that there are neural activity pattern

407 differences in how individuals acquire information in different social contexts.

\section{Acknowledgements}

We thank members of the Hofmann lab for many fruitful discussions. In particular, we

412 thank Caitlin Friesen and Isaac Miller-Crews for comments on earlier drafts of this manuscript, 413 and Nupur Shambharkar for performing the Fos cell counts. In addition, we thank Julie Butler 414 and Morgan Gustison for detailed comments on earlier drafts of this manuscript. This work was 415 supported by a UT Austin Graduate School Bruton and Summer Fellowships, and a Department 416 of Integrative Biology Doctoral Dissertation Improvement Grant (MRS); the National Science 
418 of Evolution in Action (H.A.H. and A.J.), Dr. Dan Bolnick and the Howard Hughes Medical

419 Institute (A.J.); and NSF grant IOS1354942 (HAH).

420

421

7. Author Contributions

422

MRS, AJ, and HAH designed experiments, MRS performed experiments and statistical

423 analysis, MRS and HAH wrote the manuscript, MRS, AJ, and HAH revised manuscript. 


\section{References}

1. Kendal, RL, Coolen, I, van Bergen, Y, and Laland, KN. (2005) Trade-offs in the adaptive use of social and asocial learning. Advances in the Study of Behavior, 35: 333-379.

2. Ward, AJW, Webster, MM, \& Hard, PJB. (2006) Intraspecific food competition in fishes. Fish and Fisheries, 7(4): 231-261.

3. Posadas-Andrews, A, and Roper, TJ. (1983) Social transmission of food-preferences in adult rats. Anim Behav, 31(1): 265-271.

4. Galef, BG, and Wigmore, SW. (1983) Transfer of information concerning distant foods: a laboratory investigation of the 'information-centre' hypothesis. Anim Behav, 31: 748-758.

5. Visalberghi, E, and Fragaszy, DM. (1990) Food-washing behaviour in tufted capuchin monkeys, Cebus apella, and crab eating macaques, Macaca fascicularis. Anim Behav, 40(5): 829-836.

6. Bugnyar, T, and Kotrschal, K. (2002) Observational learning and the raiding of food caches in ravens, Corvus corax: is it 'tactical' deception? Anim Behav, 64(2): 185-195.

7. Claidiere, N, Messer, EJE, Hoppitt, W, and Whiten, A. (2013) Diffusion dynamics of socially learned foraging techniques in squirrel monkeys. Curr Biol, 23(13): 1251-1255.

8. White, DJ. (2004) Influences of social learning on mate-choice decisions. Learning and Behavior, 32: 105-113.

9. Griffin, AS. (2004) Social learning about predators: a review and prospectus. Learning and Behavior, 32: 131-140.

10. Bruchey, A, Jones, CE, and Monfils, MH. (2010) Fear conditioning by-proxy: social transmission of fear during memory retrieval. Beh Brain Res, 214(1): 80-84.

11. Olsson, A, and Phelps, E. (2007) Social learning of fear. Nat Neurosci, 10(9): 1095-1102.

12. Heyes, CM. (1994) Social learning in animals: categories and mechanisms. Biol. Rev, 69: 207-231.

13. Byrne, RW. (1994) The evolution of intelligence. In Behaviour and Evolution. Cambridge, UK: Cambridge University Press.

14. Zajonc, RB. (1965) Social facilitation. Science, 149: 269-274.

15. Whiten, A, Allan, G, Devlin, S, Kseib, N, Raw, N \& McGuigan, N. (2016) Social learning in the real-world: 'Over-imitation' occurs in both children and adults unaware of participation in an experiment and independently of social interaction. PloS one, 11(7), e0159920.

16. Laland, KN and Galef, BG. (2009) The question of animal culture. Harvard University Press.

17. Laland, KN and Hoppitt, W. (2003) Do animals have culture? Evolutionary Anthropology: Issues, News, and Reviews, 12(3): 150-159.

18. Rendell, L and Whitehead, H. (2001) Culture in whales and dolphins. Behav Brain Sci, 24: 309-324.

19. Cox, J and Witten, IB. (2019) Striatal circuits for reward learning and decision-making. Nature Reviews Neuroscience, 20(8), 482-494.

20. Hoppitt, W, and Laland, KN. (2013) Social learning: An introduction to mechanisms, methods, and models. Princeton University Press.

21. Bunsey, M and Eichenbaum, H. (1995) Selective damage to the hippocampal region blocks long-term retention of a natural and nonspatial stimulus-stimulus association. Hippocampus, 5: 546-556. 
22. Alvarez, P, Lipton, PA, Melrose, R, and Eichenbaum, H. (2001) Hippocampal region on memory for a natural, nonspatial odor-odor association. Learning and Memory, 8(2): 7986.

23. Choleris, E, Clipperton-Allen, A, Phan, A, and Kavaliers, M. (2009) Neuroendocrinology of social information processing in rats and mice. Front Neuroendocrinol, 30: 442-459.

24. Amaral, DG. (2003) The amygdala, social behavior, and danger detection. Annals of the New York Academy of Sciences, 1000L 337-347.

25. Cole, AJ, Saffen, DW, Baraban, JM, \& Worley, PF. (1989) Rapid increase of an immediate early gene messenger RNA in hippocampal neurons by synaptic NMDA receptor activation. Nature, $340(6233), 474-476$.

26. Sheng, M., \& Greenberg, M. E. (1990). The regulation and function of c-fos and other immediate early genes in the nervous system. Neuron, 4(4), 477-485.

27. Bozon, B, Kelly, A, Josselyn, S, Silva, A, Davis, S, and Laroche, S. (2003) MAPK, CREB, and zif268 are all required for the consolidation of recognition memory. Phil Trans of the $R$ Soc Lon, 358(1432):805-814.

28. Loebrich, S and Nedivi, E. (2009) The function of activity-regulated genes in the nervous system. Physiol Rev 89: 1079-1103.

29. Countryman, RA, Kaban, NL, and Colombo, PJ. (2005) Hippocampal c-fos is necessary for long-term memory of a socially transmitted food preference. Neurobiol Learn Mem, 84: $175-183$.

30. Smith, CA, Countryman, RA, Sahuque, LL, and Colombo, PJ. (2007) Time-course of Fos expression in rat hippocampus and neocortex following acquisition and recall of a socially transmitted food preference. Neurobio Learn Mem, 88(1):65-74.

31. Bertaina, V and Destrade, C. (1995) Differential time courses of $c$-fos mRNA expression in hippocampal subfields following acquisition and recall testing in mice. Cogn Brain Res, 2: 269-275.

32. Tischmeyer, I and Grimm, R. (1999) Activation of immediate early genes and memory formation. CELL Mol Life Sci, 55: 564-574.

33. Ferguson, J. N., Aldag, J. M., Insel, T. R., \& Young, L. J. (2001). Oxytocin in the medial amygdala is essential for social recognition in the mouse. Journal of Neuroscience, 21(20), 8278-8285.

34. Bolhuis, JJ, Zijlstra, GGO, Den Boer-Visser, AM, Van der Zee, EA. (2000) Localized neuronal activation in the zebra finch brain is related to the strength of song learning. Proc Natl Acad Sci USA, 97, 2282-2285.

35. Bolhuis, J. J., Hetebrij, E., Den Boer $\square$ Visser, A. M., De Groot, J. H., \& Zijlstra, G. G. (2001). Localized immediate early gene expression related to the strength of song learning in socially reared zebra finches. European Journal of Neuroscience, 13(11), 2165-2170.

36. Bischof, H. J., \& Rollenhagen, A. (1999). Behavioural and neurophysiological aspects of sexual imprinting in zebra finches. Behavioural brain research, 98(2), 267-276.

37. Hofmann, HA (2003). Functional genomics of neural and behavioral plasticity. $J$ Neurobiol, 54: 272-282.

38. Maruska, KP and Fernald, RD. (2018) Astatotilapia burtoni: a model system for analyzing the neurobiology of behavior. ACS Chem Neurosci, 9: 1951-1962.

39. Hofmann, HA, Fernald, RD. (2001) What cichlids tell us about the social regulation of brain and behaviour. J Aquacult Aquatic Sci 9: 17-31. 
40. Rodriguez-Santiago, M, Nührenberg, P, Derry, J, Deussen, O, Francisco, FA, Garrison, LK, Garza, SF, Hofmann, HA, and Jordan, LA. (2020) Behavioral traits that define social dominance are the same that reduce social influence in a consensus task. Proc Nat Acad Sci of USA, 117(31): 18566-18573.

41. Fernald, RD and Maruska, KP. (2012) Social information changes the brain. Proc Nat acad Sci, 109: 17194-17199.

42. Maruska, KP, Zhang, A, Neboori, A, and Fernald, RD. (2013) Social opportunity causes rapid transcriptional changes in the social behaviour network of the brain in an African cichlid fish. J of Neuroendocrin, 25(2): 145-157.

43. O'Connell, LA, Fontenot, MR, and Hofmann, HA. (2013) Neurochemical profiling of dopaminergic neurons in the forebrain of a cichlid fish, Astatotilapia burtoni. J Chem Neuroanat, 47: 106-115.

44. Weitekamp, CA, Nguyen, J, and Hofmann, HA. (2017) Neuromolecular regulation of aggression differs by social role during joint territory defense. Integrative and Comparative Biology, 00(0):1-9. DOI: 10.1093/icb/icx009

45. O'Connell, LA and Hofmann, HA. (2011) The vertebrate mesolimbic reward system and social behavior network: a comparative synthesis. J Comp Neurol, 519(18): 3599-3639.

46. O'Connell, LA and Hofmann, HA. (2012) Evolution of a vertebrate social decisionmaking network. Science, 336(6085):1154-1157.

47. Therneau T (2020). A Package for Survival Analysis in R. R package version 3.27, https://CRAN.R-project.org/package=survival.

48. Calcagno, V and de Mazancourt, C. (2010) glmulti: an R package for easy automated model selection with (generalized) linear models. J Stat Soft, 34(12): 1-29.

49. Galef, BG and Laland, KN. (2005) Social learning in animals: empirical studies and theoretical models. Bioscience, 55(6).

50. Portavella, M, Vargas, JP, Torres, B, Salas, C. (2002) The effects of telencephalic pallial lesions on spatial, temporal, and emotional learning in goldfish. Brain Res Bull, 57: 397399.

51. Broglio, C, Gomez, A, Duran, E, Ocana, FM, Jimenez-Moya, F, Rodriguez, F, and Salas, C. (2005) Hallmarks of a common forebrain vertebrate plan: specialized pallial areas for spatial, temporal and emotional memory in actinopterygian fish. Brain Research Bulletin, 66: 277-281.

52. Elliott, SB, Harvey-Girard, E, Giassi, ACC, and Maler, L. (2016) Hippocampal-like circuitry in the pallium of an electric fish: possible substrates for recursive pattern separation and completion. J Comp Neurol, 525(1):8-46.

53. Portavella, M, Torres, B, and Salas, C. (2004) Avoidance response in goldfish: emotional and temporal involvement of medial and lateral telencephalic pallium. J Neurosci, 24(9): 2335-2342.

54. Rodriguez, F, Lopez, JC, Vargas, JP, Gomez, Y, Broglio, C, \& Salas, C. (2002) Conservation of spatial memory function in the pallial forebrain of reptiles and ray-finned fishes. Journal of Neuroscience, 22(7).

55. Salas, C., Broglio, C., \& Rodríguez, F. (2003). Evolution of forebrain and spatial cognition in vertebrates: conservation across diversity. Brain, behavior and evolution, 62(2), 72-82.

56. Davis, M. (1994). The role of the amygdala in emotional learning. In International review of neurobiology (Vol. 36, pp. 225-266). Academic Press. 
57. Gentile, C. G., Jarrell, T. W., Teich, A., McCabe, P. M., \& Schneiderman, N. (1986). The role of amygdaloid central nucleus in the retention of differential Pavlovian conditioning of bradycardia in rabbits. Beh Brain Res, 20(3), 263-273.

58. LeDoux, J. E. (2000). Emotion circuits in the brain. Annual review of neuroscience, 23(1), 155-184.

59. Lee, T., \& Kim, J. J. (2004). Differential effects of cerebellar, amygdalar, and hippocampal lesions on classical eyeblink conditioning in rats. Journal of Neuroscience, 24(13), 3242-3250.

60. Wullimann, M. F., \& Mueller, T. (2004). Teleostean and mammalian forebrains contrasted: Evidence from genes to behavior. The Journal of comparative neurology, 475(2), 143.

61. Yamamoto N, Ishikawa Y, Yoshimoto M, Xue HG, Bahaxar N, Sawai N, Yang CY, Ozawa H, Ito H. 2007. A new interpretation on the homology of the teleostean telencephalon based on hodology and a new eversion model. Brain Behav Evol 69:96104.

62. Braford MR Jr. 2009. Stalking the everted telencephalon: comparisons of forebrain organization in basal ray-finned fishes and teleosts. Brain Behav Evol 74:56-76.

63. Nieuwenhuys R. 2011. The development and general morphology of the telencephalon of actinopterygian fishes: synopsis, documentation and commentary. Brain Struct Funct 215:141-157.

64. Alunni A, Blin M, Deschet K, Bourrat F, Vernier P, Re'taux S. 2004. Cloning and developmental expression patterns of Dlx2, Lhx7 and Lhx9 in the medaka fish (Oryzias latipes). Mech Dev 121:977-983

65. Demski LS, Knigge KM. 1971. The telencephalon and hypothalamus of the bluegill (Lepomis macrochirus): evoked feeding, aggressive and reproductive behavior with representative frontal sections. J Comp Neurol 143:1-16.

66. Clayton, DF. (2000) The genomic action potential. Neurobiol Learn Mem, 74: 185-216.

67. Clayton, DF, Anreiter, I, Aristizabal, M, Frankland, PW, Binder, EB, and Citri, A. (2019) The role of the genome in experience-dependent plasticity: extending the analogy of the genomic action potential. Proc Nat Acad Sci USA, doi.org/10.1073/pnas.1820837116.

68. Fernald, RD and Hirata, NR. (1977) Field study of Haplochromis burtoni: quantitative behavioural observations. Animal Behaviour, 25.

69. Maguire, S, DeAngelis, R, Dijkstra, PD, Jordan, A, and Hofmann, HA. (2021) Social network dynamics predict hormone levels and behavior in a highly social cichlid fish. Hormones \& Behavior, in revision.

70. Jones, CE, Monfils, MH. (2016) Dominance status predicts social fear transmission in laboratory rats. Anim Cogn, 19: 1051-1069.

71. Berridge KC, Robinson TE (1998) What is the role of dopamine in reward: hedonic impact, reward learning, or incentive salience? Brain Res Brain Res Rev 28:309-369.

72. Heimovics, SA, Cornil, CA, Ball, GF, and Riters, LV. (2009) D1-like dopamine receptor density in nuclei involved in social behavior correlates with song in a context-dependent fashion in male European starlings. Neuroscience, 159: 962-973.

73. Schultz, W., Apicella, P. \& Ljungberg, T. (1993) Responses of monkey dopamine neurons to reward and conditioned stimuli during successive steps of learning a delayed response task. J Neurosci. 13:900-913. 
610

611

612

613

614

615

616

617

618

619

620

621

622

623

624

625

626

627

628

629

630

631

632

633

634

635

636

637

638

639

74. Schultz, W. (2006). Behavioral theories and the neurophysiology of reward. Annu. Rev. Psychol., 57, 87-115.

75. Messias, JPM, Paula, JR, Grutter, AS, Bshary R, and Soares, MC. (2016) Dopamine disruption increases negotiation for cooperative interactions in a fish. Scientific Reports, 6:2-9.

76. Weitekamp, CA, Nguyen, J, and Hofmann, H. (2017) Social context affects behavior, preoptic area gene expression, and response to D2 receptor manipulation during territorial defense in a cichlid fish. Genes, Brain and Behavior, 16(6):601-611.

77. Messias, JPM, Santos, TP, Pinto, M and Soares, MC. (2016) Stimulation of dopamine D1 receptor improves learning capacity in cooperating cleaner fish. Proc $R$ Soc B Biol Sci, 283(1823):20152272.

78. Rodriguez-Santiago, $M$ and Hofmann, HA. (2021) Social instability during group formation and learning does not impact the beahvioral or neural response to an association task. In preparation.

79. Goodson, J. L. (2013). Deconstructing sociality, social evolution and relevant nonapeptide functions. Psychoneuroendocrinology, 38(4), 465-478.

80. Goodson, J. L., \& Bass, A. H. (2001). Social behavior functions and related anatomical characteristics of vasotocin/vasopressin systems in vertebrates. Brain Research Reviews, 35(3), 246-265.

81. Semsar, K, Kandel, F, and Godwin, J. (2001) Manipulations of the AVT system shift social status and related courtship and aggressive behavior in the Bluehead Wrasse. Hormones and Behavior, 40(1): 21-31.

82. Insel, T, Wang, Z, and Ferric, C. (1994) Patterns of brain vasopressin receptor distribution associated with social organization in microtine rodents. J Neurosci, 14(9): 5381-5392.

83. Goodson, J. L., \& Wang, Y. (2006). Valence-sensitive neurons exhibit divergent functional profiles in gregarious and asocial species. Proceedings of the National Academy of Sciences, 103(45), 17013-17017.

84. Baeyens, D and Cornett, L. (2006) The cloned avian neurohypophysial hormone receptors. Comp Biochem \& Phisio B, 143(1): 12-19.

85. Popik, P and Van Ree, JM. (1993) Social transmission of flavored tea preferences: facilitation by a vasopressin analog and oxytocin. Beh and Neur Biol, 59: 63-68.

86. Choleris, E and Kavaliers, M. (1999) Social learning in animals: sex differences and neurobiological analysis. Pharm Biochem and Beh, 64(4): 767-776. 


\section{Figure Captions}

641

642 Figure 1. Learning rate is faster in a social context. A) Comparison of the cumulative

643 response probability to a visual cue discrimination task between a social and a non-social context

644 shows that groups have a higher response probability than individuals in a non-social context (p

$645=0.004)$. B) Although the rate of response probability is significantly different between social

646 contexts, the total number of trials required to achieve this response criterion is not statistically

647 different between contexts $(\mathrm{p}=0.426)$.

648

649

Figure 2. Principal component analysis (PCA) of neural activity shows differential expression pattern with learning context. A) Scatter plot of all Fos expression data separates out by social condition (social, non-social) across PC1. B) Vector plot showing the PCA variables that load on PC1 and PC2. C) Plot showing the percent of the variance explained by each PC. PC1 (D-F) and PC2 (G-I) loadings plotted across trials based on social condition (D and $\mathrm{G})$ and whether learning response was reached $(\mathrm{E}$ and $\mathrm{H})$. Boxplot showing that PC1 loadings (F) differentiates data by social condition but not by learning response while PC2 loadings (I) differentiate do not differentiate context across learning response.

657

658

Figure 3. Neural activity across brain regions varies over trials and with learning. Fos

659 expression was quantified as a marker of neural activity in the Dlg, Dm-1, and Vs regions of the 660 forebrain (A, D, F). In the Dlg, there was a significant increase in activity from trial 6 to 14 in the social context, while there was no difference in activity across trials in the non-social context

662 (B). Neural activity was significantly highest in learners in the social context (C). In the Dm-1, 663 activity significantly decreased over trials (E). Activity was significantly highest in the Dm-1 in 664 the social context when learning had not occurred (F). In the Vs, activity significantly increased 665 after trial 6 in the social context $(\mathrm{G})$ and was significantly higher in the social context with 666 learning $(H)$. 
